the first time, and in the other 38 she either had an unreduced occipito-posterior presentation, a much scarred and undilatable perineum, the child's head was unusually large, or the after-coming head required rapid delivery to prevent stillbirth. In none of them did any untoward symptoms arise; the patient was unaware either of the incisions being made or of the sutures being passed. usually use a pair of stout, blunt-pointed scissors, such as is employed in cutting the umbilical cord.

I do not purpose here to enter into the history of the operation; this has already been thoroughly worked out by Wilcox. ${ }^{1}$ The steps of the operation, with careful drawings of the direction of the incision, are admirably described and figured by Dickinson. ${ }^{2}$ Parvin ${ }^{3}$ was one of the earliest advocates of the operation in America.

Brook-street, W.

\section{THE NITROGEN BALANCE IN DIABETES MELLITUS AND ITS IMPORTANCE IN TREATMENT.}

\section{BY P. J. CAMMIDGE, M.D. LOND., M.R.C.S.,} L.R.C.P. LOND.

IT has long been recognised that the essential feature of diabetes mellitus is a failure of carbohydrate metabolism, with the consequent presence of sugar in the urine. As the metabolic changes have been more thoroughly and completely investigated it has been increasingly apparent, however, that a simple defect in the utilisation of carbohydrates is comparatively rare and that there is usually more or less interference with the utilisation of fats and proteins as well. These defects are relatively slight in some instances, while in others they are prominent and constitute the most serious feature of the case; but it is obvious that if the maximum benefit is to be derived from the dietetic treatment of the condition all deviations from the normal must be taken into account, otherwise a diet may be prescribed which in the end will do more harm than good. The failure of a "carbohydrate-free" diet to control the disease, or even to check the glycosuria in some cases, is thus frequently accounted for, since it attempts to deal with only one feature of the case, the defective utilisation of carbohydrates, and neglects other possible deficiencies in metabolism. With the discovery of the acetone bodies, and the realisation of their significance as a sign of faulty fat metabolism, an important advance was made on Rollo's plan of excluding carbohydrates as much as possible from the diet in all cases of glycosuria, and it is now common knowledge that the persistent presence of acetone bodies in the urine is an indication that an ordinary "strict diet" cannot be tolerated. The occurrence of defects in the utilisation of proteins and the results of increasing the protein content of the diet beyond the patient's powers of dealing with them efficiently are, unfortunately, not so well known, although they are equally important. One reason for this neglect undoubtedly is that the products of imperfect protein metabolism cannot be recognised in the urine by an easily applied colour-test like sugar, acetone, or aceto-acetic acid, but are only to

1 The Operation of Episiotomy, New York Medical Journal, 1885, p. 178, with literature.

with literature. be detected by making a series of quantitative analyses of the collected and measured 24 hours' excretion when the patient is on a diet of known composition. As a similar series is needed for the accurate determination of the carbohydrate tolerance of the case, it only entails a little extra work on the part of the observer however.

The simplest and most easily obtained criterion of the state of protein metabolism is the nitrogen balance-that is to say, the relation between the intake of nitrogen in the food and the total nitrogen output in the urine. The former may be estimated from food tables, the latter is best determined by Kjeldahl's process or some modification of it. The method used in my laboratory is carried out as follows. One cubic centimetre of the mixed urine is carefully measured by means of an accurate pipette into a Jena glass flask or large test-tube, 2.5 c.c. of pure concentrated sulphuric acid and four drops of platinic chloride solution (1 per cent.) are added, and the mixture is heated in a fume chamber, or with the flask connected to Folin's fume-absorber, until it is colourless (usually about half an hour). The flask is then cooled and its contents are washed out into an aspiration tube (B) (Fig. 1) with about 20 c.c. of

FIG. 1.

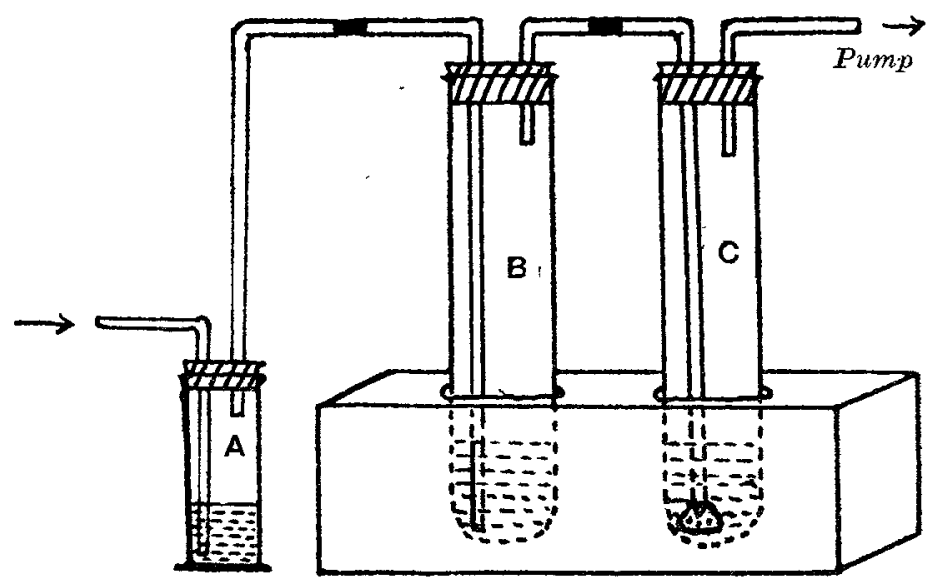

water. The tube is afterwards cooled and about 5 c.c. of paraffin oil are poured in. Having ready a rubber stopper provided with the necessary con. nexions 20 c.c. of sodium hydrate solution (30 per cent.) are added, the tube is then corked and quickly connected to a receiver (c) containing 10 c.c. of decinormal sulphuric acid. The inlet branch of the aspiration tube is joined to a wash-bottle containing strong sulphuric acid (A) and the outlet branch of the receiver to a suction pump. A current of air is drawn through the apparatus for an hour, slowly at first and then more quickly. At the end of that time the apparatus is disconnected and the acid in the receiver titrated with $\mathrm{N} / 50$ sodium hydrate solution, using alizarin red as the indicator and continuing until the solution turns red, not violet. The difference between the reading obtained and the amount of acid placed in the receiver (calculated on the same basis as the strength of the sodium hydrate) multiplied by 0.028 gives the percentage of nitrogen. Thus if 12 c.c. of soda solution were used the difference between this and the amount of acid in the receiver would be 38 (for 10 c.c. of decinormal acid is equivalent to 50 c.c. of $\mathrm{N} / 50$ acid, and $50-12=38$ ). Multiplying by $0^{\circ} 028$ we have $1^{\circ} 064$ per cent. of nitrogen. This multiplied by the decilitres of urine passed in the day (e.g., 1200 c.c.) gives the total nitrogen output in the urine (e.g., $1.064 \times 12=12 \cdot 768$, or, in round figures, 
12.8 grammes). The advantages of this method are that it is economical of labour and time if several specimens are to be examined and when the aspiration process for estimating true ammonia nitrogen and amino-acid nitrogen ${ }^{1}$ and the urease method for determining urea ${ }^{2}$ are employed, since they can be run in series on the same pump. To allow of the body completing the catabolism of the protein as well as the carbohydrate of each day's diet the collection of the urine should be arranged so that the longest possible interval intervenes between the last meal and the finish of the 24 hours period. It is usually best to start the collection with the first specimen voided after breakfast and finish with the last passed before that meal next day in order that at least 12 hours without food will elapse before the collection is completed.

In a healthy individual the total urinary nitrogen is practically equal to the amount ingested, barring the small extra-renal excretion in the fæces, perspiration, hair, and nails, which averages about 1 grammedaily. The level of nitrogenous equilibrium varies with the character of the diet, although there is always a tendency to establish a correspondence between the intake and the output-in spite of wide variations in the nature of the food. A similar tendency is seen in diabetics, but their powers of adaptation are diminished, and in advanced cases may be very defective. A highly protein diet raises the output of nitrogen in the urine as in a healthy person, but the increase is often out of proportion to the intake and remains so, or even tends to increase, as long as the excess is continued. A diminution of the protein, on the other hand, will frequently bring down an excessive excretion of nitrogen in the urine more than can be accounted for by the lessened intake of nitrogen in the food. This is probably to be accounted for by an increased susceptibility of the diabetic to the specific dynamic effect of protein, with a consequent tendency to abnormal tissue waste, owing to the defective utilisation of carbohydrates. The addition of protein foods to the diet of diabetics frequently fails, therefore, to accomplish its aim, and instead of improving nutrition only raises the level of metabolism and promotes tissue waste. In cases where the utilisation of sugar is very defective an increase in the glycosuria also results and the excretion of oxybutyric acid and acetone bodies rapidly rises, owing to the inability of the tissues to oxidise the sugar and fatty acids derived from the non-nitrogenous portion of the aminoacids formed from the ingested and broken.down tissue proteins. The prognosis in cases where the output of nitrogen in the urine regularly exceeds the intake in the food is always serious, for unless nitrogenous equilibrium can be established loss of weight and strength must continue, while the tendency will be for the glycosuria and acidosis to increase. Systematic reduction of the protein intake, guided by daily analyses of the urine, often suffices to control the condition, but rest in bed, with freedom from excitement and worry, is sometimes also necessary. The free use of opiates may be required occasionally. In my own practice I now very rarely use preparations of opium except in severe cases where nitrogenous equilibrium cannot be established by any other means.

The following case (Fig. 2) illustrates in a striking manner the results that can be obtained by regulating the diet according to the nitrogen balance.

1 Cammidge: The Lavcet, Nov, 8th, 1913, p. 1319.

2 Marshall : Journal of Biological Chemistry, vol. Xiv.. 1913, p. 283; rol. xv., 1913, pp. 487 and 495 .
The patient, a naval officer aged 24, was advised to consult me by Mr. P. S. B. Wetherall, of Weymouth. When I saw him on July 24th, 1915, he stated that, although he had suffered from heartburn and some abdominal discomfort occasionally for two or three years, his general health had been good until Christmas, 1914, when he noticed that he was very thirsty and easily tired. His urine was examined and sugar

FIG. 2.

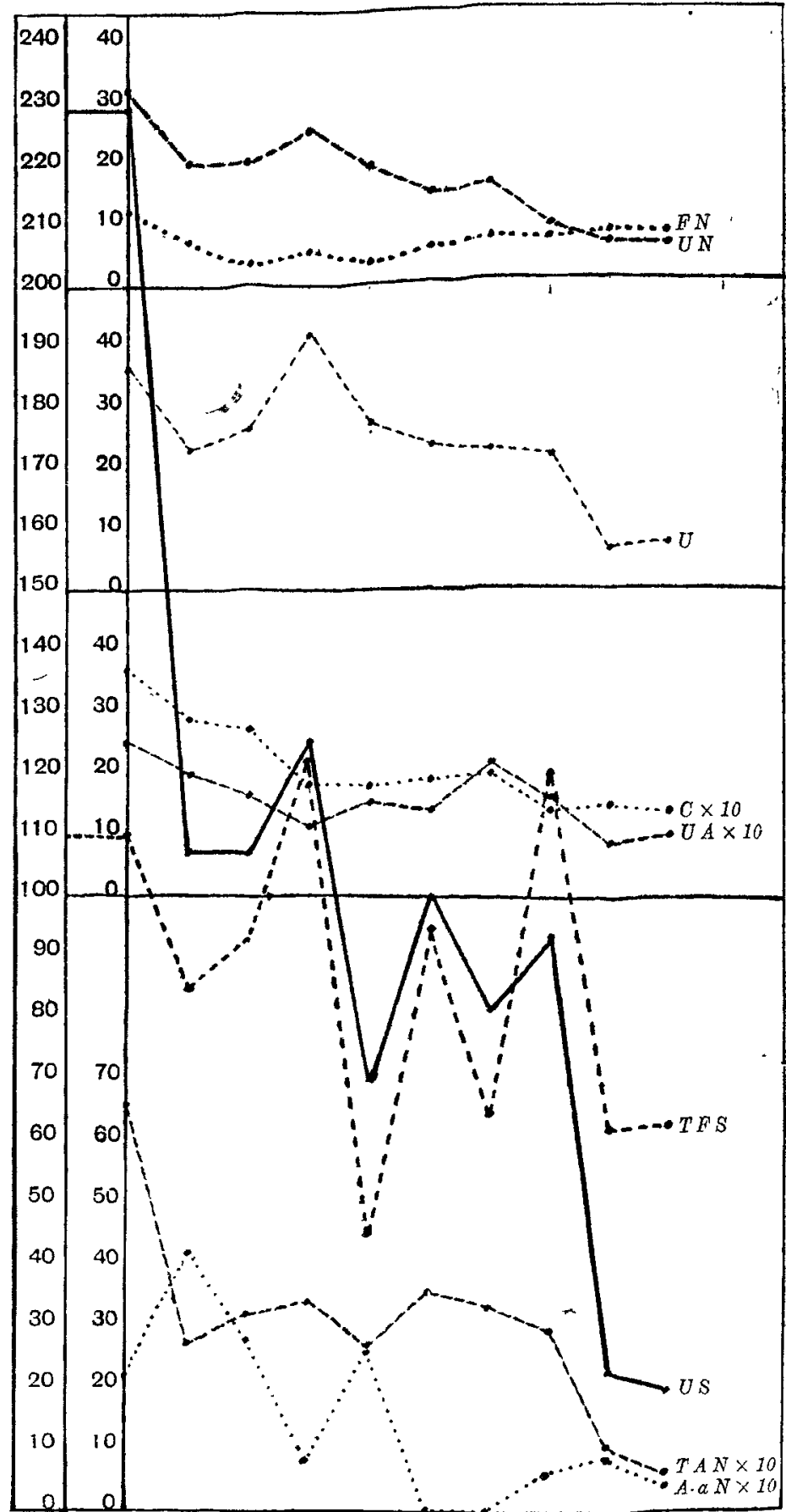

$F N$, Food nitrogen. $U N$, Urinary nitrogen. $U$, Urea. $C \times 10$, Creatinin $\times 10$. UA $U A$, Uric acid $\times 10 . T F S$, Total food sugar. $U S$, Urinary sugar. $T A N \times 10$, True ammonia nitrogen $\times 10$. $A-a N \times 10$, Amino-acid nitrogen $\times 10$.

was found. Under treatment by an "anti-diabetic" diet, containing as much meat and other protein foods as he cared to take and with a limited allowance of carbohydrate, his thirst had diminished, but he steadily lost weight and was obviously getting weaker. Physical examination showed nothing of note except a patch of xanthoma diabeticorum on each forearm. His blood was found to contain 0.33 per cent. (?) of sugar, but as there was marked lipæmia, which interfered with accurate estimation, it was probably higher. His alveolar air contained 3.4 per cent of carbon dioxide, as compared with the normal of $5-6$ per cent., showing that there was considerable acidosis. I took him into a nursing home and put him to bed at once. On a diet containing 73 grammes of protein (12 grammes of nitrogen) and 52 grammes of carbohydrate, with a total sugar value of 110 grammes, allowing for the sugar which could be formed from the protein, a 24 hours collection of 
the urine measured 5270 c.c. and contained 229 grammes of sugar (4.35 per cent.), $31 \cdot 8$ grammes of nitrogen, $19 \cdot 7$ grammes of beta-oxybutyric acid, 1.05 grammes of acetone bodies, 6.6 grammes of ammonia nitrogen, 1.05 grammes of calcium, $0 \cdot 84$ gramme of magnesium, $2 \cdot 2$ grammes of aminoacid nitrogen, $36 \cdot 4$ grammes of urea, $2 \cdot 5$ grammes of uric acid, 3.74 grammes of creatinin, and 8.4 grammes of phosphates. There was also half a gramme of albumin, and microscopical examination showed numerous hyaline and finely granular casts. Altogether the case was apparently as hopeless as it could be, short of actual coma, which seemed imminent. It was evident that the patient was passing more sugar in his urine than could be accounted for by the carbohydrate, or even the protein, in the diet and that he was forming sugar from his own tissues. The nitrogen balance tended to confirm this conclusion, and with the markedly abnormal excretions of amino-acids, uric acid, creatinin, \&c., pointed to there being serious tissue waste and defective protein metabolism. That a similar inability to metabolise fats, as well as carbohydrates and proteins, existed was shown by the lipæmia and the high output of oxybutyric acid, while the excess of ammonia nitrogen, calcium, and magnesium pointed to there being serious acidosis. The protein of the diet was reduced to 47 grammes ( 7 grammes of nitrogen) and then to 20 grammes ( 3 grammes of nitrogen), but the sugar value was kept at nearly the same level, with the result that the sugar excretion promptly fell to less than half its former value (viz., to 107 grammes), and the total nitrogen dropped over twice the amount corresponding to the reduction in the food nitrogen (viz. to 20 grammes) At the same time the oxybutyric acid came down to 6.2 grammes and with a marked fall in the ammonia nitrogen, calcium, and magnesium showed that there was a considerable improvement in the acidosis. The protein intake was then slightly raised and more carbohydrate was given, but as the urinary nitrogen and sugar excretions promptly rose, although not nearly to their former level, both were again reduced with a resulting further fall. A second attempt to increase the nitrogenous intake, and later the amount of carbohydrate in the diet, was more successful, giving rise to only a temporary rise in the sugar excretion and causing no appreciable increase in the urinary nitrogen. The latter then steadily fell until finally nitrogenous equilibrium was established, an intake of 7 grammes of nitrogen in the food being represented by an output of 5 grammes in the urine. At the same time the quantity of urine was reduced, ranging about 2000 c.c., and the sugar excretion came down to 20 grammes for the 24 hours. The albumin completely disappeared and no casts could be found. Examination of the blood now showed 0.2 per cent. of sugar, with only very slight lipæmia. The alveolar carbon dioxide had risen to 4.6 per cent., and the oxybutyric acid excretion had fallen to 3.6 grammes.

Since the patient returned home I have heard that his strength has much improved and that he is able to take a fair amount of exercise without fatigue. He is bright and cheerful, and his present condition is in marked contrast to his desperate state three months ago.

Abnormalities of the nitrogen balance are not confined to advanced and serious cases, but may exist when the urine contains only a small amount of sugar and when the glycosuria has been controlled by an "anti-diabetic" diet. Loss of strength, irritability of temper, nervous distubances, neuralgic pains, and complaints of difficulty in keeping warm are the commonest subjective symptoms. Diminution in weight is a more certain indication, but is apt to be a fallacious guide unless found to be progressive in character. As an example of this type I may quote a case brought to me in January, 1912, by Dr. J. D. R. Munro, of Nantwich.

The patient was a lady, aged 37, who had been found to be suffering from glycosuria the previous September, when her urine contained 2 per cent. of sugar. Seen by a well-known London physician, she was put on an "anti-diabetic" diet of the usual type. For a time she improved, but in November she complained of being depressed and miserable, and it was found that she was losing weight. A second consultation was arranged therefore. She was then advised to adhere to the diet that had been previously prescribed and to go abroad for the winter. This she refused to do, although she was warned that her only hope lay in wintering in a warmer climate. When I first saw her, in January, 1912, she was so emaciated that it was painful for her to sit or lie for long in one position, and she stated that she had lost $18 \mathrm{lb}$. in weight in the previous three months. Her urine contained only a trace of sugar, but there was a well-marked reaction for acetone and aceto-acetic acid. A new diet was given her, but as she did not improve very much $I$ advised her to come into a nursing home in order that her metabolism might be thoroughly investigated. After some delay it was arranged. On a diet containing 70 grammes of carbohydrate, 97 grammes of protein (15 grammes of nitrogen), with a total sugar value of 148 grammes, she passed 2600 c.c. of urine containing $9 \cdot 6$ grammes of sugar and 24 grammes of nitrogen. The nitrogen content of the diet was reduced to 13 grammes and the urinary nitrogen fell to 20 grammes. A further reduction of the food nitrogen to 10 grammes was followed by a drop in the nitrogen of the urine to 8 grammes, 1 gramme less than the intake when allowance was made for an extra-renal excretion of 1 gramme. Meanwhile the sugar excretion had also dropped in spite of the fact that the quantity of carbohydrate in the diet was kept at about the same level. At the same time the acetonæmia diminished and only faint traces of acetone could be detected in the urine. For a few days the patient's weight remained stationary, but when she left the home she was $2 \frac{1}{2} \mathrm{lb}$. heavier than when she came in. On the diet which was then prescribed the increase in weight continued and by the middle of December, 1912, she was 1 st. $10 \mathrm{lb}$. heavier than when I first saw her 11 months before. Subsequently the weight has remained constant save for slight variations in either direction probably due to accidental causes. Nitrogenous equilibrium has been well maintained ever since it was first established, even when more protein was slowly and cautiously introduced into the diet. When the patient left the home her urine was free from any trace of sugar, and although there has been some glycosuria at times it has responded to readjustment of the diet.

I think there can be little doubt that this patient, like many others, was the victim of a rule-of-thumb method of treatment based upon empirical findings obtained over 100 years ago, for as soon as her diet was arranged on lines in accordance with modern research she improved rapidly and, as she says, "life became worth living." At any rate, she is now in comparatively good health and is able to follow her ordinary occupations in comfort and with enjoyment, four years after she had been warned that her span of life was likely to be measured by months rather than by years.

Nottingham-place, $w$.

\section{OPHTHALMIC NOTES IN A GENERAL HOSPITAL IN EGYPT.}

BY C. WYNN WIRGMAN, M.J.LonD., F.R.C.S.ENG., IIEUTENANT, R.A.M.o. (T.).

For two months I was in charge of the ophthalmic wards in the hospital to which I was attached in Egypt, and during that time about 100 ophthalmic cases came under my care, besides ordinary wounds, as the wards could not be entirely reserved for the former. A few notes on the ophthalmic cases may be interesting as showing the range of work.

Cases sent up to the hospital from convalescent camps constituted an out-patient clinic, from which any case requiring special attention was admitted. Of cases directly from the front the great majority naturally were injuries, while from the convalescent camps refractive errors were in the majority. In many of the latter it was a matter of surprise that they had ever been passed fit for service, as the defects of vision were so gross that the most casual examination could not have 